\title{
Comparison of Different Image Enhancement Methods for Effective Whole-Body Bone Scan Image
}

\author{
Sulieman Mohammed Salih Zobly \\ Department of Physics \& Medical Instrumentation, University of Gezira, Medani, Sudan
}

Email address:

sulieman16@gmail.com

\section{To cite this article:}

Sulieman Mohammed Salih Zobly. Comparison of Different Image Enhancement Methods for Effective Whole-Body Bone Scan Image. Advances in Bioscience and Bioengineering. Vol. 7, No. 3, 2019, pp. 55-59. doi: 10.11648/j.abb.20190703.16

Received: June 14, 2019; Accepted: July 23, 2019; Published: August 28, 2019

\begin{abstract}
Nuclear medicine is one of the most important diagnostic tools used for different types of investigations such as a thyroid scan, renal function and whole-body bone scan. The main problem in nuclear medicine imaging system is the resulting images degraded with large amounts of noise. In this work we want use four different enhancement methods to enhance wholebody bone scan image so as to reduce the noise from the image and improve the resolution to achieve a better image quality and maintain quality for accurate diagnosis. Histogram equalization, adaptive histogram equalization, log transformation and gamma correction algorithms were used to improve the image quality. Four pair of bone scan images from gamma camera were used to perform this work. Enhanced images were quantified and evaluated by calculating the Peak Signal Noise Ratio, Mean Square Error and entropy. The result shows that the gamma correction algorithm gives best result among the four algorithms used for enhancing the bone images. The gamma correction algorithm can assist the radiologist in diagnosis the patient and quantify any changes accurately and quickly.
\end{abstract}

Keywords: Whole-body Bone Scan, Gamma Camera, Image Enhancement, Nuclear Medicine

\section{Introduction}

The nuclear medicine imaging system used to provide information on a wide range of disease after injecting the patient with a very small amount of radionuclide. The radionuclide imaging is one of the most important applications of radioactivity in nuclear medicine. It used to obtain a picture of the distribution of a radioactivity within the body [1]. The importance of nuclear medicine imaging lies in its ability to provide an exquisitely sensitive measure of a wide range of biologic processes in the body [1]. The most popular imaging system used in nuclear medicine is the gamma camera. It's a device used to image gamma radiation emitting from the radioisotope. The gamma camera imaging system has been used for different types of investigation, such as heart imaging, thyroid scans, whole-body bone scan and other organ function [2, 3]. The most important and useful application of gamma camera is the whole-body bone scan, which is used to identify the abnormalities in the bone and how metastases respond to radiotherapy or chemotherapy treatment. Also, bone scan is very important tool for detecting wither the cancer spread over the skeleton started from different organs.

When some features need to be viewed the quality of the image need to be improved, this process known as image enhancement. Image enhancement is one of the most important tools used in image process to modify the original image so the resulting image is more suitable for specific application [4, 5]. In medical imaging, many images suffer from noise and low contrast, there for its necessary to enhance the image before displaying for visual, detail worth full and accurate diagnosis. Therefore, there are many enhancement techniques presented to obtain satisfactory results, the techniques can be divided into two board categories; spatial domain methods and frequency domain methods [4]. In this paper only Histogram equalization, adaptive histogram equalization, log transformation and gamma correction algorithms will be discussed and used for enhancing whole-body bone scan image. Histogram equalization has been applied for different application such as contrast enhancement and chest $\mathrm{x}$-ray image enhancement $[6,7]$. Histogram equalization is one of the most important techniques based on spatial domain processing, which can be used effectively to extract images details. Adaptive histogram 
equalization algorithm is very effective for images contain local regions of low contrast bright or dark regions. This algorithm has been applied for enhancing the images contrast [8]. Log transformation is useful algorithm for stretching image contrast [5]. Urvashi and Yojana show in their work [9] it's possible to used log transform algorithm to extract the details from the images efficiently. Gamma correction is one of the most important pre-processing steps in medical imaging processing methods. Many enhancement algorithms have been developed based gamma correction such as automatic medical image contrast enhancement based on gamma correction, and Gaussian noise reduction [10, 11].

In this study we intend to use different enhancement algorithms to enhanced whole-body bone scan images so as to reduce the noise from the resulting images beside enhance the images details and maintain the image quality. Among these algorithms we want to select the more suitable algorithm which can improve the image and maintain the image quality.

The rest of paper organized as follow, in section II the algorithms used to enhance the images in this work explained in details beside how the bone scan image performed in nuclear medicine. The result of enhanced images was explained and discussed in section III. In the last section the conclusion of the work was presented.

\section{Material and Methods}

In this section we will discuss the bone scan imaging in nuclear medicine and the four enhancement methods used to improve the image quality in this work.

\subsection{Bone Scan}

A whole-body bone scan image is one of the most important diagnostics in nuclear medicine, which is helping the physician to diagnose different disease. The bone scan used for detecting bone injury or bone infection, which in undetectable on X-ray imaging systems.

The whole-body bone scan was acquired using a SPECT gamma camera (Mediso, Sprit Nucline) three to four hours after injecting the patient with radiopharmaceutical (Tc$99 \mathrm{~m})$. The patient lies down supine on the scanning bed and the camera detectors face each other's while whole-body images are obtained. This generally takes about 20 minutes, although the time may be shorter or slightly longer depend on the type of the gamma camera used [12]. The dual head camera was used to record anterior and posterior whole-body bone scan with resolution 256 x 1024 pixels. Four patients were used to obtain eight pairs of images.

\subsection{Histogram Equalization (HE)}

Histogram equalization is one of the most important techniques for image quality improvement and is the most important part for any image processing. HE used to improve the visual appearance of the images and can be applied for whole image or to a part of the image [5].
The HE, is the transform function $\mathrm{T}$ transform the input intensity, $\Upsilon$ into a new intensity value, $\ddot{\Upsilon}$. The transform function is the product of the accumulated histogram and scale factor. The new intensity value, fitted to the range of the intensity value via scale factor. This can be presented as follows:

$$
\gamma^{\prime}=\mathrm{T}(\gamma)=\sum_{\mathrm{i}=0}^{\gamma} \mathrm{n}_{\mathrm{i}} \frac{\text { max int ensity }}{\mathrm{N}}
$$

Where $\mathrm{n}_{i}$ is the number of pixel at intensity $i, \mathrm{~N}$ is the total of pixel in the image.

This means that the transform generates an image with intensity levels are equally and cover the entire range $[0,1]$. The transform function is nothing more than the cumulative distribution function. The histogram of the image will not be equal due to the discrete value of the nature of the variables [4].

\subsection{Adaptive Histogram Equalization (AHE)}

Adaptive histogram equalization (AHE) is image processing, computer based technique used to improve image contrast. HE generates only one histogram whereas AHE methods compute several histograms corresponding to a district section of the image and uses what to redistribute the intensity value of the image [13].

\subsection{Power-law Operator}

It is defined as follows:

$$
\mathrm{S}=\mathrm{Cr}^{\lambda}
$$

Where $\mathrm{C}$ and $\lambda$ are positive constant, $\mathrm{r}$ is the value of input pixel and $\mathrm{S}$ is the corresponding value of the output pixel.

The effect of this operator can be controlled by changing the gamma value. In power-law a wider range of output values can be mapped with a narrow range of dark values. The phenomena of using power-law to enhance image, is known as a gamma correction. The gamma correction is an important tool for enhancing and displaying an image accurately for better diagnosis. The image quality depends on the gamma value, which leads to changes in brightness of the image [14].

\subsection{Log Transformation}

Is one of the useful enhancement methods, which is operated in a simple point processor, each pixel value is replaced with its logarithm. Log transform maps a narrow range of low gray level value into a wider range. The general form of $\log$ transform is:

$$
S=C \log (r * 1)
$$

Where $\mathrm{C}$ is a constant and it assumed $\mathrm{r} \geq 0$.

The quality of resulting images was evaluated by using three different performance measures, root mean square error (RMSE), peak signal to noise ratio ((PSNR) and entropy. 
Root mean square error is one of the most important performance measure used to evaluate the image performance. The RMSE calculated for two images I and II with dimension of (m-by-n), where I is the original image and II is the reconstructed image. The RMSE measurement is easily computed by the square root of (mean square error MSE) the average squared difference between every pixel in recovering image and the original image. The RMSE calculated as follows:

$$
\begin{gathered}
\operatorname{MSE}=\frac{1}{n m} \sum_{i=0}^{m} \sum_{j=0}^{n 1}[I(i, j) \quad I I(i, j)]^{2} \\
\operatorname{RMSE}=\sqrt{\mathrm{MSE}}
\end{gathered}
$$

Where, I (i, j) and II $(i, j)$ are the pixel values of the original and recovered image respectively and $m, n$ are the size of an image.

PSNR reflects the differences of the information contained between an original and recovered image. The PSNR numbers are reported in Decibels $(\mathrm{dB})$ as a measure of the relative weight between two images. A higher number in $\mathrm{dB}$ indicates a higher correlation. The PSNR is directly proportional to the image quality. When PSNR is higher this indicates that the reconstruction is of higher quality. PSNR calculated as follows:

$$
\mathrm{PSNR}=20 \log _{10}(255 / \mathrm{RMSE}) \mathrm{dB}
$$

Image entropy is a quantity which is used to describe the amount of information which must be coded in the image. An image with very low entropy near to zero is perfect.

The image entropy can be calculated as follows:

$$
\text { entropy }=\sum_{i} p_{i} \log _{2} p_{i}
$$

Where $\mathrm{P}_{\mathrm{i}}$ is the probability that the difference between 2 adjacent pixels is equal to $i$, and $\log _{2}$ is the base 2 logarithm.

\section{Result and Discussion}

The whole-body bone scan images, which acquired by using SPECT gamma camera (Mediso, Sprit Nucline) three to four hours after injecting the patient with radiopharmaceutical (Tc-99m) were enhanced with selected enhancement methods and evaluated with three different performance measures. Figures 1, 2, 3, and 4 shows the result of applying a selected enhancement method to four different patient bone scan images.

Figure 1 show the image of the first patient with metastasis, figure $1 \mathrm{a}$ is the original image and b, c, d, and e are images enhanced with $\mathrm{HE}$, AHE, Gamma correction and log transformation algorithms respectively. From figures gamma correction and log transformation gives clear images with less noise on the other hand HE and AHE images have a very high noise and the legion point are not clear in the resulting images. The log transformation subtracts the noise from the image and remains the legion. The problem of this algorithm, small legion can be subtracted from the resulting image. The gamma correction algorithm removes most of the noise and remains legion clear, which is made the image clear and easy to be diagnosed with the physician.

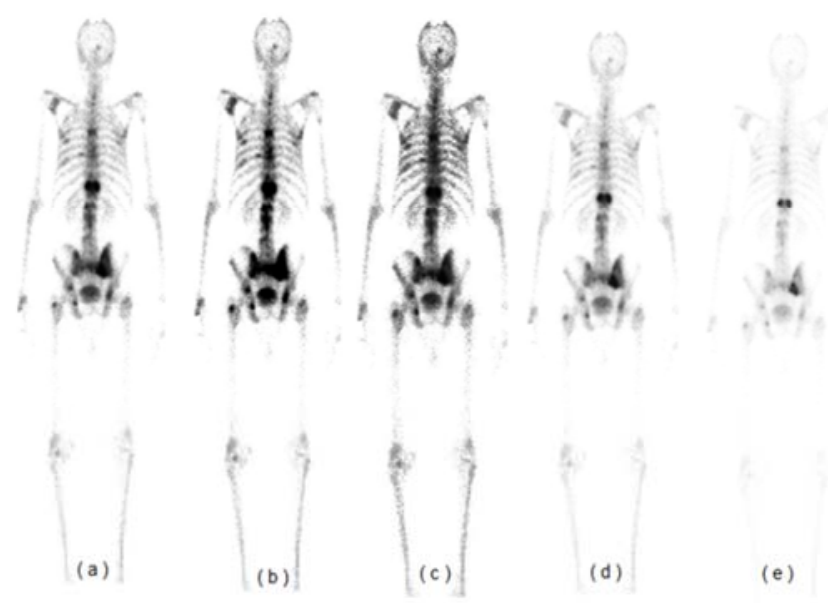

Figure 1. First patient images, (a) original image, (b) image enhanced with $H E$, (c) image enhanced with AHE, (d) image enhanced with gamma correction and (e) image enhanced with log transformation.

Figure 2 show images of the second patient used to perform this study. Figure 2a represents the original image and figure 2. B, c, d, and e are enhanced images with HE, AHE, gamma correction and log transform respectively. AHE algorithm gives image with very high noise compare to the other algorithms and gamma correction gives image with very low noise and clear, which is made the diagnosis easy for the physician. Log transform give image with noise free, but small legion can be subtracted from the enhanced image.

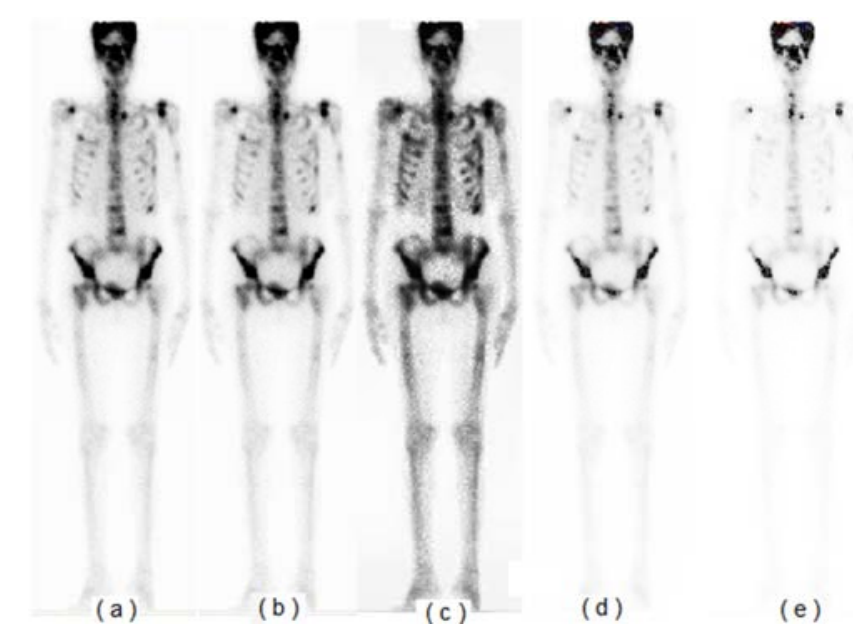

Figure 2. Second patient images, (a) original image, (b) image enhanced with HE, (c) image enhanced with AHE, (d) image enhanced with gamma correction and (e) image enhanced with log transformation.

Figure 3 show images of the second patient used to perform this study. Figure 3 a represents the original image and figure 3. B, c, d, and e are enhanced images with HE, AHE, gamma correction and log transform respectively. Compared to the original image gamma correction gives 
clearer image with less noise. Also log transformation algorithm gives a clear image without any noise.

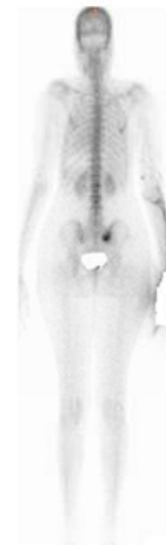

(a)

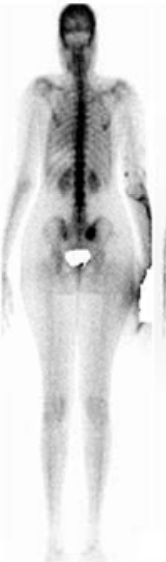

(b)

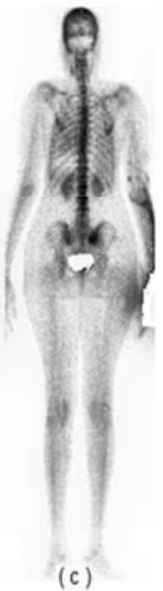

(d)

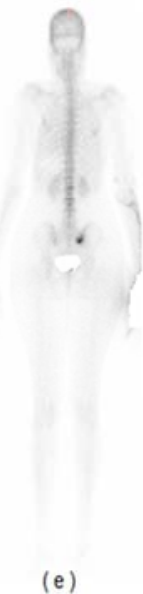

(e)
Figure 3. Third patient images, (a) original image, (b) image enhanced with $H E$, (c) image enhanced with AHE, (d) image enhanced with gamma correction and (e) image enhanced with log transformation.

Figure 4 show images of the fourth patient used to perform this study. Figure $4 \mathrm{a}$ represents the original image and figure 4. B, c, d, and e are enhanced images with HE, AHE, gamma correction and $\log$ transform respectively. The images enhanced with $\mathrm{HE}$ and AHE are very noisy, this noise reduced gradually by using gamma correction and $\log$ transform enhancement algorithms. Log transformation gives clear image and gamma correction clear image with a few noises.

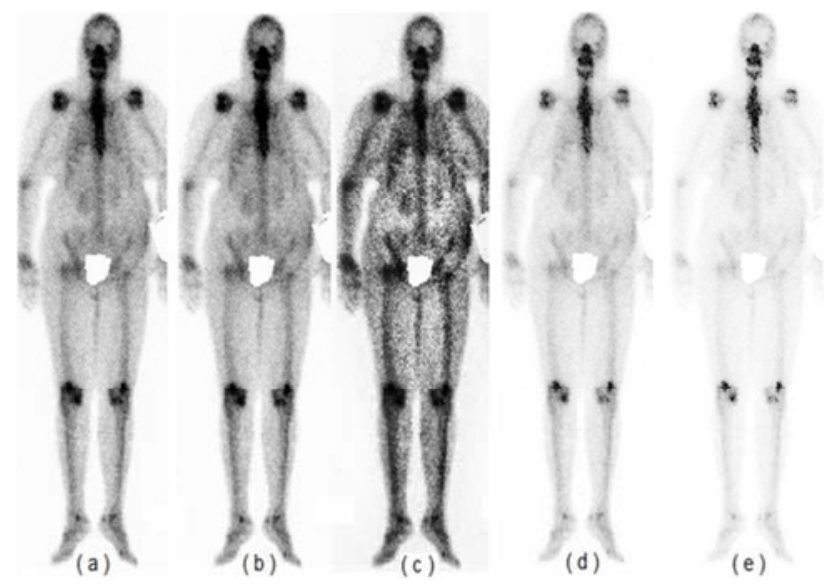

Figure 4. Fourth patient images, (a) original image, (b) image enhanced with $H E$, (c) image enhanced with AHE, (d) image enhanced with gamma correction and (e) image enhanced with log transformation.

Zobly et al [14] shows that the bone can be enhanced with gamma correction and gives clear image but this result depend on the gamma value as gamma value increased or decreased the image will be degraded. Acording to his study the most suitable gamma value was 0.6. Some experiment done for selecting a suitable gamma value the result shows that an image enhanced with gamma value of 0.6 was the best and this result was the same of that shown by Zobly. Aslantas et al [15] used feature extraction and classification to detect the hotspot in the body. His result shows that the algorithm Identify 120 out of 130 images. This algorithm can only detect the hotspot areas in the bone and can't improve the quality of the image as shown in our result. Zobly and Elfadil [16] they show it's possible to enhance bone scan with the most common enhancement algorithms but the quality of the resulting image varies according to the algorithm. X. Liu [17] proposed an algorithm based on fuzzy membership to enhance the image. He divide the image into two regions high gray level and low gray level, but this algorithm applied to normal images only.

The resulting images quality measured by using three different performance measures, RMSE, PSNR and entropy. Table I shows the result of the performance measure. The result shows that the RMSE was very low from images enhanced with log transform and AHE gives higher RMSE in all enhanced images. RMSE from images enhanced with gamma correction is lower than that gain from images enhanced with HE. PSNR also was calculated from the enhanced images, the result shows that $\log$ transforms gives higher PSNR and AHE gives lower PSNR, in the other hand log transform gives better image. The gamma correction algorithm enhanced image better than that enhanced with HE. Beside RMSE and PSNR the entropy was calculated. A log transforms image has low entropy compare to the other algorithm. The entropy of the image enhanced with gamma correction is lower than the entropy of the image enhanced with HE. AHE gives higher entropy among all the algorithms used for whole body bone scan image.

We can conclude that the image enhanced with log transform is the best, gives lower RMSE, lower entropy and higher PSNR. On the other hand this algorithm can discard the enhanced images, thus the gamma correction algorithm is better than log transform because the resulting image is free of noise and give very clear legion.

Table 1. The performance measure of the images.

\begin{tabular}{llllll}
\hline Images & $\begin{array}{l}\text { Quality } \\
\text { measue }\end{array}$ & HE & AHE & Log & Gamma \\
\hline \multirow{3}{*}{ First } & RMSE & 9.888 & 11.468 & 3.699 & 4.283 \\
& PSNR & 28.2286 & 26.9406 & 36.7675 & 35.4944 \\
& Entropy & 3.043 & 3.398 & 1.832 & 2.550 \\
& RMSE & 10.871 & 19.434 & 2.484 & 4.310 \\
Second & PSNR & 27.4054 & 22.3591 & 40.2268 & 35.4399 \\
& Entropy & 5.316 & 6.686 & 3.407 & 4.458 \\
& RMSE & 18.582 & 19.098 & 0.7368 & 2.709 \\
Third & PSNR & 22.7489 & 22.5107 & 50.7842 & 39.4724 \\
& Entropy & 5.688 & 6.308 & 4.101 & 4.443 \\
& RMSE & 14.058 & 18.944 & 2.456 & 4.826 \\
& PSNR & 25.1719 & 22.5812 & 40.3237 & 34.4583 \\
& Entropy & 6.268 & 7.241 & 4.502 & 5.472 \\
\hline
\end{tabular}

\section{Conclusions}

The four enhancement methods have been applied to whole body bone scan image generated from the gamma camera by using Tc-99m. The result shows that the whole body bone scan image quality can be improved with enhancement methods for better visualization and accurate diagnosis. AHE gives lowest 
PSNR among algorithm used in this work and log transform give higher value, which is mean that log transform image was the best. The quality of the images enhanced with gamma correction methods was better than that enhanced with HE. The entropy calculated from the image enhanced with $\log$ transform was 3.7, 3.4, 4.1 and 4.5 from the first, second, third and fourth image respectively, AHE gives higher value of entropy form all the images group. Beside the PSNR and entropy the RMSE was calculated from all the enhanced images The RMSE form images enhanced with AHE was the highest and that enhanced with log transform are the lowest. From the result we can conclude that $\log$ transform gives an image with noise free and clear details, which is helping the radiographer in diagnosis. However, the problem of this algorithm sometime during enhancement some important details can be removed from the image. Gamma correction also gives a very clear image without any noise and very clear legion. This can help the physician to diagnose the images accurately. HE and AHE give a noisy image after enhancement. Gamma correction method is the best enhancement algorithm this algorithm can be used for enhancing whole body bone scan image and lead to accurate diagnoses and an image with low noise.

\section{Conflict of Interest}

The author declares that there is no conflict of interest regarding the publication of this paper.

\section{References}

[1] S. Cherry, J. Sorenson, M. Phelps, physics in Nuclear Medicine, $3^{\text {rd }}$ Edition, Elsevier 2000.

[2] J. Mallard, M. Myers, clinical application of gamma camera, physics in medicine and biology, vol. 8 (2).

[3] A. Kantzas, et al., Application of gamma camera imaging and SPECT systems in chemical processes chemical engineering journal, vol. 77 (1-2), 2000, pp: 19-25.

[4] R. Gonzalez, R. Woods, Digital Image Processing, second Edition, Prentice Hall, Inc, 2002.

[5] R. Gonzalez, R. Woods, Steven L. Eddins, Digital Image Processing using MATLAB, 2end Edition, Gatesmark Publishing, 2009.
[6] D. Chang, W. Wu, Image contrast enhancement based on a histogram transformation of local standard deviation, IEEE transaction on medical imaging, vol. 17 (4), 1998, pp. 518531.

[7] R. Senthilkumar, M. Senthilmurugan, TRIAD histogram to enhance chest X-ray image, international journal of advanced research in computer and communication engineering, vol. 3 (11), 2014, pp. 8577-8580.

[8] J. A. Stark, W. J. Fitzgerald, An Alternative Algorithm for Adaptive Histogram Equalization, Graphical Models and Image Processing, vol. 58 (2), 1996.

[9] Urvashi Manikpuri, Yojana Yadav, Image Enhancement through Logarithmic Transformation, international journal of innovation research in advanced engineering, 2014, 357-362.

[10] S. Suman, et al. Image enhancement using geometric mean filter and gamma correction for WCE images, ICONIP, 2014, pp. 276-283.

[11] K. Somasundaram, P. Kalavath Medical Image Contrast Enhancement based on Gamma Correction 2014.

[12] M. Ouvrier, S. Vignot, J. Thariat. [State of the art in nuclear imaging for the diagnosis of bone metastases]. Bull Cancer. 2013 Nov. 100 (11): 1115-1124. [Medline].

[13] D. Sonker, Comparison of Histogram Equalization Techniques for Image Enhancement of Grayscale images in Natural and Unnatural Light, international journal of engineering research and development, Vol. 8 (9), 2013, pp. 57-61.

[14] S. Zobly et al, Selecting Suitable Gamma Value for Bone Scan Image Enhancement using Gamma Correction Method, Red Sea University Journal of Basic \& Applied science, vol. 2 (1), 2017, pp. 485-490.

[15] A. Aslantas et al, A computer-aided Diagnosis System for Whole Body Bone Scintigraphy Scans, J Can Res Ther 2016, pp. 87-92.

[16] Zobly S. and Elfadel M., Whole-Body Bone Scan Image Enhancement Algorithms, 2018 International Conference on Computer, Control, Electrical and Electronic Engineering (ICCCEEE), Khartoum, 2018, pp. 1-4.

[17] X. Liu, An Improved Image Enhancement Algorithm Based on Fuzzy Set, 2012 International Conference on Medical Physics and Biomedical Engineering, 2012, 790-797. 